\title{
Stage III Renal Pelvis Cancer AJCC v7
}

National Cancer Institute

\section{Source}

National Cancer Institute. Stage III Renal Pelvis Cancer A/CC v7. NCI Thesaurus. Code C6146.

Stage III includes: T3, NO, M0. T3: Tumor invades beyond muscularis into peripelvic fat or the renal parenchyma. NO: No lymph node metastasis. MO: No distant metastasis. (AJCC 7th ed.) 